\title{
Endophytic Fungi and Ecological Fitness of Chestnuts
}

\author{
Rosario Nicoletti $^{1,2} \mathbb{D}$, Gabriele Loris Beccaro ${ }^{3} \mathbb{D}$, Agnieszka Sekara ${ }^{4}$, Chiara Cirillo ${ }^{2, *(D)}$ \\ and Claudio Di Vaio ${ }^{2}$ (D) \\ 1 Research Center for Olive, Fruit and Citrus Crops, Council for Agricultural Research and Economics, \\ 81100 Caserta, Italy; rosario.nicoletti@crea.gov.it \\ 2 Department of Agricultural Sciences, University of Naples Federico II, 80055 Portici, Italy; \\ claudio.divaio@unina.it \\ 3 Department of Agricultural, Forest and Food Sciences, University of Turin, 10095 Grugliasco, Italy; \\ gabriele.beccaro@unito.it \\ 4 Department of Horticulture, University of Agriculture, 31-425 Krakow, Poland; agnieszka.sekara@urk.edu.pl \\ * Correspondence: chiara.cirillo@unina.it
}

check for updates

Citation: Nicoletti, R.; Beccaro, G.L.; Sekara, A.; Cirillo, C.; Di Vaio, C. Endophytic Fungi and Ecological Fitness of Chestnuts. Plants 2021, 10, 542. https://doi.org/10.3390/ plants10030542

Academic Editor: Luigi De Masi

Received: 23 February 2021

Accepted: 11 March 2021

Published: 13 March 2021

Publisher's Note: MDPI stays neutral with regard to jurisdictional claims in published maps and institutional affiliations.

Copyright: (c) 2021 by the authors. Licensee MDPI, Basel, Switzerland. This article is an open access article distributed under the terms and conditions of the Creative Commons Attribution (CC BY) license (https:// creativecommons.org/licenses/by/ $4.0 /)$.

\begin{abstract}
Chestnuts (Castanea spp.) are plants of relevant economic interest in the agro-sylvicultural contexts of mountain regions throughout the temperate zone, particularly in the northern hemisphere. In recent years, several biological adversities have repeatedly endangered species belonging to this genus, calling for coordinated actions addressed to contrast their decline. These actions have mainly focused on the control of key pests/pathogens and the improvement of resistance/tolerance by the plant host, while the role of microorganisms as mediators of interactions between plants and the noxious agents has been less considered, essentially by reason of a limited knowledge on their ecological impact. In line with the increasing awareness of the basic importance of microbial symbionts in regulating plant fitness in both natural and crop contexts, this paper offers an overview on the occurrence and effects of endophytic fungi of chestnuts.
\end{abstract}

Keywords: Castanea spp.; defensive mutualism; endophytes; mycorrhizae; plant growth promotion

\section{Introduction}

Chestnuts (Castanea spp.) are geographically distributed in three main areas throughout the world (Asia, Europe, North America) where they have an invaluable cultural heritage but also an important economic and environmental role in many agroforestry systems. Their nuts provided for centuries a dietary staple in rural areas and, when dried, a stored food for the whole year; the wood is still today used as firewood or building timber.

The genus Castanea ( $x=12,2 n=24)$, chestnuts and chinkapins, belongs to the Fagaceae (Cupuliferae) family, which includes 6 genera (Castanea, Quercus, Castanopsis, Fagus, Nothofagus, Lithocarpus) and approximately 1000 species. It is closely related to the genus Castanopsis [1], is widespread in the boreal hemisphere and encompasses 13 species. The natural distribution of the European or sweet chestnut (C. sativa) covers Europe and some Mediterranean countries. In China, Japan, Vietnam, North and South Korea C. mollissima, C. crenata, C. seguinii, C. henryi occur. In North America, C. dentata is still present only along the Appalachian Mountains, due to the devastating impact of the chestnut blight. Castanea pumila is found in the southeastern United States [2,3].

Castanea species show different ecological and morphological traits, vegetative habit, wood and nut characteristics, pedoclimatic adaptability, resilience and resistance to biotic and abiotic stresses, reflecting the adaptation of this genus to different environmental conditions [4]. The main cultivated species are C. sativa, C. mollissima and C. crenata, due to their large nut size, and marroni cultivars (C. sativa) are considered the most commercially valuable. Castanea sativa and $C$. dentata are also cultivated for timber production, whilst many interspecific hybrids are used for nut production or as rootstocks [5]. 
Chestnut cultivation has been affected by several problems that brought to a dramatic collapse of the whole production system for many years, particularly in Europe and North America. Most of the mountain populations in the European countries were dependent on chestnut farming, both for timber and for fruits (over $1 \mathrm{mln}$ tons of nuts per year at the beginning of the 20th century) [6]. The progressive introduction of new pests and diseases caused massive damages to $C$. sativa and $C$. dentata cultivations. Moreover, the industrial era and the introduction of more remunerative crops (potatoes, cereals) caused the abandonment of large chestnut growing areas. However, over the past 15 years the global world chestnut production has been growing even with some fluctuations, mainly due to Chinese production increase, reaching approximately $2.3 \mathrm{mln}$ tons of fresh fruits in 2019 over more than 600,000 hectares. Eastern Asia and Mediterranean Europe are the two main production areas supplying, respectively, almost $90 \%$ and over $7 \%$ of the chestnuts produced worldwide. The intense scientific and agronomic activity promoted in the last decades of the 20th century by the Chinese government provided strong tools for improving chestnut cultivation. The increase in global chestnut production was driven both by the Chinese policies and by the global population growth and popularity of healthy eating. These key drivers are expected to continue promoting growth of the chestnut crop in the future [3].

\section{Ecological and Technical Features of Chestnut Orchards}

The ecological features of chestnut orchards, their agronomic management and architecture strongly depend on species and country. The best soils for chestnut are deep, soft, volcanic in origin, and rich in phosphorus, potassium, and organic matter. The soil $\mathrm{pH}$ should be in the range 5.0-6.5; therefore, soils with active limestone are not optimal, because Castanea species are very sensitive to high $\mathrm{pH}$ values. Soil permeability is very important. In fact, the crop performs better in well-drained, loam to sandy-loam soils, while heavy, washed out, clayey, stagnant soils which favor root rot must be avoided [6].

Chestnut tolerates cold winters and requires average temperature of $8-15^{\circ} \mathrm{C}$, with a monthly average of $10^{\circ} \mathrm{C}$ for at least six months. Castanea sativa is more cold-resistant $\left(-15\right.$ to $\left.-20^{\circ} \mathrm{C}\right)$ than the Euro-Japanese hybrids. Despite late bud-break (March-April in the Northern Hemisphere), the trees may be prone to spring frosts which damage young shoots. Temperatures of $27-30{ }^{\circ} \mathrm{C}$ are necessary during pollination. European and Japanese cultivars require about $800-900 \mathrm{~mm}$ /year of rainfall, well distributed during the growing season, while Euro-Japanese hybrids and C. mollissima are much more waterdemanding (1200-1300 mm/year). In temperate climates, sweet chestnut should not be planted above 800-900 m, while for hybrids the maximum altitude is about 500-600 m [5].

Ecological features of a chestnut orchard can be influenced by many natural and anthropogenic parameters, such as soil, climate, rootstock, cultivar, cover crops, irrigation system and fertilization [7]. Concerning soil, an increase in the organic matter content is often obtained through sheep and cattle pasture, outside the harvesting period, or with the use of manure (10-15 tons/ha/year). Mown grass, leaves, husks and the small pruned material are often left on the ground as they provide further enrichment in organic matter. Fertilization of the orchard is every year carried out according to soil conditions and uptakes [8]. Biological or conventional management are both adopted by chestnut growers and could strongly influence the crop ecological features.

The orchard model, traditional or intensive, could also strongly influence the crop ecological features. Traditional orchards are mainly located in mountainous areas while intensive orchards are a relatively recent practice and are usually located in lowlands, quite far from the typical ecological conditions of the species [9]. For all species, except in traditional European chestnut orchards, the general trend is to increase plant density to develop in a relatively short time maximum bearing per unit area. Plantation density can affect the orchard microclimatic conditions and can range from 100 to 550 trees/ha, based on species, variety, genotype-environment interactions and cultural practices. For traditional plantations of C. sativa, spacing ranges from 8-10 $\mathrm{m}$ apart in rows and 8-12 $\mathrm{m}$ between 
rows. For the most vigorous Euro-Japanese hybrids the distances range between $7 \times 8 \mathrm{~m}$ (178 trees/ha) and $8 \times 10 \mathrm{~m}$ (125 trees/ha). Castanea sativa and the Euro-Japanese hybrids can be cultivated in high density plantations $(3 \mathrm{~m} \times 10 \mathrm{~m})$. For $C$. crenata distances of about $5 \times 7 \mathrm{~m}$ (285 trees/ha, in deep fertile soils) or $7 \times 7 \mathrm{~m}$ (204 trees/ha) are recommended. Castanea mollissima, grown in China and the Unites States, is managed in a high or semi-high density scheme, due to the smaller tree size. Planting patterns may be rectangular, squared or triangular, but the first scheme is mostly used because of easier management [3]. Nowadays, many traditional plantations need renewal and recovery after years of abandonment or following the attacks of pests and diseases that have compromised their efficiency.

\section{The Relevance of Microorganisms in the Management of Chestnuts}

Besides climatic and agronomic aspects, fitness and productivity of chestnuts are remarkably influenced by the manifold interactions established with different kinds of microorganisms. As in most forest trees which are commercially exploited in both natural and anthropized contexts, members of this component of biodiversity occur systematically and play several functional roles on both the roots and the above-ground plant parts. It can be said that they ultimately produce notable direct and indirect effects on the economy of the resident communities in the areas where chestnuts shape ecosystems, landscape and farming activities.

Fungi undoubtedly represent the main group of microbes associated with chestnut plants, variously influencing their fitness. A basically topographic difference separates species associated with roots, many of which pertain to the well-known category of the mycorrhizae. Ectomycorrhizal fungi (EMF) are well-documented as symbionts of Castanea spp. [10], abundantly covering the smallest branchings of a root system which is strong, expanded and penetrates the soil deeply. EMF provide incontestable beneficial effects to the chestnut orchard ecosystem. In fact, they stimulate release of nutrients from the soil sorption complex, making them available to the host plant. The constant access to water and mineral resources makes trees more resilient to biotic and abiotic stresses. Indeed, the biochemical cross-talk between fungi and host plants shapes the relationship and increases fitness of both symbionts. Reactive oxygen species may be essential initial products for regulating interactions during the early stages of EMF establishment and signalling molecules for symbiosis establishment. In this regard, oxidative bursts were observed two hours after inoculation of the model EMF Pisolithus tinctorius on C. sativa roots, followed by increased superoxide dismutase and catalase activities in root cells [11].

EMF change in composition with the age of the trees, shaping ecological succession. Mature trees can host up to 46 EMF species [12], while 39 EMF genera were collected from root tips of 100-year-old chestnut [13]. The young chestnut trees are hosts of "earlystage" EMF, such as Scleroderma spp., Laccaria spp. and Cenococcum geophilum. The easy dispersal and fast colonization capacity by these fungi on young root systems, even in stressful ecological conditions, can be suitable for nursery inoculation [14,15]. Late-stage EMF, generally dominant in mature stands, comprise Amanita, Boletus, Cantharellus, Cortinarius, Lactarius, Russula, and Tricholoma species, which are of particular interest as valuable culinary ingredients and can provide additional profits to local communities [16]. The Russulaceae members are among the EMF associated with the residual natural stands of C. dentata in North American forests [17]. Moreover, successful trials have been carried out demonstrating the ability by truffle species (Tuber aestivum, T. uncinatum, and T. brumale) to colonize roots of $C$. sativa. The development of commercial inoculation protocols with these species is of high economic relevance [18].

Hypogeous EMF surveys provide valuable information on the reproductive investment by chestnut-related symbiotic fungi. However, these trials partially reflect the soil fungal diversity and interspecies relations in the chestnut rhizosphere [19]. The cited study showed approximately 35\% discontinuity in the fungal species composition between the above and below ground methods of sampling of EMF in C. dentata. Of the 46 sequences identified on root tips, only 16 represented the above ground mushroom survey. Among 
the EMF identified molecularly from root tips of Castanea $\times$ coudercii [20], 13 species had not been previously documented as symbionts of chestnuts, including Hymenogaster sp., Thelephora sp., Tomentella sp.

In the early 20th century, research on EMF of C. sativa and C. dentata was highly disturbed by large-scale mortality of trees due to the spread of fungal diseases [21]. Both American and sweet chestnut represent the main components of the respective forest ecosystems, shaping their ecological associations. Considering that their extinction could have triggered widespread environmental disturbances, an extensive reintroduction project was realized involving disease resistant hybrids of $C$. sativa with $C$. crenata and $C$. mollissima, or plants grafted on hybrid rootstocks [22]. Hybrid and grafted chestnuts, which exhibit better performance, could develop individual EMF associations, diversity and colonization rates. In this respect, a previously cited study [20] determined the presence of 9 orders, 15 families, 19 genera and 27 species of EMF fungi on Castanea $\times$ coudercii, most of them generalist, early-stage species. Scleroderma spp. were the most abundant, while C. geophilum was found in most of the trees but colonized a small fraction of root tips. Although the authors did not confirm differences in EMF diversity between grafted and non-grafted specimens, the results could concurrently improve the management of chestnut agroecosystems and increase the production of mushrooms. In another study Castanea hybrids had similar interactions with soil biota to their parent species, $C$. dentata, and may fill a similar below-ground niche [23]. However, non-native pathogen presence in restoration sites can affect growth and survival of Castanea hybrids. Colonization by Cortinarius spp. facilitated the survival of native C. dentata infected by Cryphonectria sp. [24], and inoculation with Hebeloma crustuliniforme decreased disease symptoms in C. sativa infected by Phytophthora cambivora [25]. The cited research highlights that natural mutualistic interactions may be effective in the control of chestnut pathogens.

\section{Occurrence of Endophytic Fungi in Chestnuts and Ecological Implications}

Although characterized by synchronized development inside the plant tissues and nutrient transfer at the interfaces [26], EMF are not included in the category of endophytes since at some extent they also grow saprophytically in the soil. Conversely, the definition of endophytes, on which this paper is more specifically centered, does not reflect a specialized nutritional function, and it is conventionally applied to microorganisms that colonize living, internal tissues of plants without causing any immediate, overt negative effect [27].

Data concerning occurrence of endophytic fungi of chestnuts (Table 1) are only available from a low number of countries, indicating that consideration for the ecological implications and the economic impact related to this component of biodiversity is still quite limited. Possibly connected with a higher importance as an economic crop in Europe, most investigations have been carried out on $C$. sativa, with 76 taxa reported so far $(2 / 3$ of which identified at the species level), while the species C. crenata, C. dentata and C. mollissima appear to have been less frequently investigated, basically in Japan, the United States and China. As a general aspect, identifications concerning stem (shoots, branches, etc.) refer to the occurrence of endophytes in subcortical tissues, while no findings were reported from xylem $[28,29]$.

In some cases, conditions of detection did not fully meet the basic requirements referring to the true endophytic condition. In fact, a recent study based on ITS metabarcoding was addressed to establish whether fungal communities within cynipid galls are different from foliar endophytes. Unfortunately, results were basically presented with reference to classes to which the detected OTUs belong, and identifications at the species level was only carried out for the main OTUs, without distinguishing their origin (gall or leaf) [30]. Therefore, entries in Table 1 referring to this study must be taken with circumspection. Moreover, some uncertainty is entailed in a few reports concerning endophytic fungi associated with cankers produced by $C$. parasitica on both $C$. sativa and C. dentata [31-36]. Even if in these cases the plant material used for isolations was not asymptomatic following infection by a known pathogen, the sterilization procedure 
ensured that at least the isolated fungi had colonized the plant tissues before sampling and were not epiphytic contaminants.

Table 1. Endophytic fungi reported from Castanea spp.

\begin{tabular}{|c|c|c|c|}
\hline Endophyte $^{1}$ & Plant Part & Country & Reference \\
\hline \multicolumn{4}{|c|}{ Castanea sativa } \\
\hline Acremonium cf. curvulum & shoot (phellem) & Bellinzona, Switzerland & [28] \\
\hline \multirow{4}{*}{ Alternaria alternata } & several plant parts & Eurobin and Monbulk, Australia & [37] \\
\hline & shoot & Ticino, Switzerland & [38] \\
\hline & bud & Ankara, Turkey & [39] \\
\hline & bark & Vinhais, Portugal & {$[40]$} \\
\hline \multirow{3}{*}{ Alternaria sp. } & shoot & Geneve and Ticino, Switzerland & [41] \\
\hline & leaf or gall & Southern Tuscany, Italy & [30] \\
\hline & bud & Ankara, Turkey & [39] \\
\hline Apiognomonia errabunda & leaf & Cureglia and Zarei, Switzerland & {$[42]$} \\
\hline Arcopilus aureus * & bark & Vinhais, Portugal & {$[40]$} \\
\hline Arthrinium arundinis & leaf & Vejoris, Spain & [43] \\
\hline \multirow{2}{*}{ Aspergillus sp. } & stem & Black Sea region, Turkey & {$[31]$} \\
\hline & bud & Ankara, Turkey & [39] \\
\hline Asterosporium sp. & shoot (phellem) & Bellinzona, Switzerland & [28] \\
\hline Aureobasidium pullulans & shoot & Ticino, Switzerland & {$[38,41]$} \\
\hline Aureobasidium sp. & bud & Ankara, Turkey & [39] \\
\hline Biscogniauxia mediterranea & bark & Valpaços and Vinhais, Portugal & [40] \\
\hline \multirow{2}{*}{ Botryosphaeria dothidea } & shoot & Ticino, Switzerland & [41] \\
\hline & leaf or gall & Southern Tuscany, Italy & [30] \\
\hline Botryosphaeria sp. & several plant parts & Eurobin and Monbulk, Australia & [37] \\
\hline Botryotinia pelargonii & leaf or gall & Southern Tuscany, Italy & {$[30]$} \\
\hline Botrytis cinerea & several plant parts & Eurobin and Monbulk, Australia & [37] \\
\hline Chaetomium sp. & several plant parts & Eurobin and Monbulk, Australia & {$[37]$} \\
\hline Cladosporium cladosporioides & several plant parts & Eurobin and Monbulk, Australia & [37] \\
\hline \multirow{2}{*}{ Cladosporium sp. } & fruit & Eurobin and Monbulk, Australia & [37] \\
\hline & bud & Ankara, Turkey & [39] \\
\hline \multirow{3}{*}{ Colletotrichum acutatum } & shoot (phellem) & Bellinzona, Switzerland & [28] \\
\hline & leaf, shoot & Monti Cimini, Italy & {$[44]$} \\
\hline & leaf & Vejoris, Spain & [43] \\
\hline Coprinellus domesticus & bark & Oghuz, Azerbaijan & [33] \\
\hline Coryneum modonium & shoot (phellem) & Bellinzona and Murg, Switzerland & [28] \\
\hline \multirow{3}{*}{ Cryphonectria parasitica } & shoot (phellem) & Bellinzona, Switzerland & [28] \\
\hline & sprout & Fossemagne, France & {$[45]$} \\
\hline & bark & Valpaços and Vinhais, Portugal & [40] \\
\hline Cytospora chrysosperma & bark & Valpaços, Portugal & [40] \\
\hline Cytospora diatyrpelloidea & bark & Valpaços and Vinhais, Portugal & [40] \\
\hline Cytospora eucalypticola & bark & Valpaços, Portugal & {$[40]$} \\
\hline Cytospora quercicola & bark & Vinhais, Portugal & [40] \\
\hline \multirow{3}{*}{ Dendrostoma castaneum $^{*}$} & shoot (phellem) & Bellinzona and Murg, Switzerland & [28] \\
\hline & fruit & Toricella, Switzerland & [46] \\
\hline & branch & Astroni Nature Reserve, Italy & [47] \\
\hline
\end{tabular}


Table 1. Cont

\begin{tabular}{|c|c|c|c|}
\hline Endophyte $^{1}$ & Plant Part & Country & Reference \\
\hline Diaporthe amygdali & bark & Oghuz, Azerbaijan & [33] \\
\hline Diaporthe eres & shoot & Geneve and Ticino, Switzerland & {$[38,41]$} \\
\hline Diaporthe foeniculina & branch & Astroni Nature Reserve, Italy & [48] \\
\hline Diaporthe sp. & $\begin{array}{l}\text { shoot (phellem) } \\
\text { leaf or gall } \\
\text { bud }\end{array}$ & $\begin{array}{c}\text { Bellinzona and Murg, Switzerland } \\
\text { Southern Tuscany, Italy } \\
\text { Ankara, Turkey }\end{array}$ & $\begin{array}{l}{[28]} \\
{[30]} \\
{[39]}\end{array}$ \\
\hline Diplodia seriata & leaf or gall & Southern Tuscany, Italy & [30] \\
\hline Diplodina castaneae * & $\begin{array}{l}\text { shoot (phellem) } \\
\text { stem, twig } \\
\text { stem } \\
\text { bark, stem }\end{array}$ & $\begin{array}{c}\text { Bellinzona and Murg, Switzerland } \\
\text { Chablais and Ticino, Switzerland } \\
\text { Northern Spain } \\
\text { Ismailly, Qabala, Sheki } \\
\text { (Azerbaijan) }\end{array}$ & $\begin{array}{l}{[28]} \\
{[48]} \\
{[48]} \\
{[33]}\end{array}$ \\
\hline Epicoccum nigrum & $\begin{array}{l}\text { several plant parts } \\
\text { bark } \\
\text { bud }\end{array}$ & $\begin{array}{c}\text { Eurobin and Monbulk, Australia } \\
\text { Balakan, Azerbaijan } \\
\text { Ankara, Turkey }\end{array}$ & $\begin{array}{l}37] \\
{[33]} \\
{[39]}\end{array}$ \\
\hline Eutypella sp. & bark & Zagatala, Azerbaijan & [33] \\
\hline Fusarium ciliatum & leaf or gall & Southern Tuscany, Italy & [30] \\
\hline Fusarium lateritium & leaf or gall & Southern Tuscany, Italy & [30] \\
\hline Fusarium oxysporum & leaf or gall & Southern Tuscany, Italy & {$[30]$} \\
\hline Fusarium sp. & $\begin{array}{l}\text { fruit } \\
\text { leaf or gall } \\
\text { leaf } \\
\text { bud }\end{array}$ & $\begin{array}{c}\text { Eurobin and Monbulk, Australia } \\
\text { Southern Tuscany, Italy } \\
\text { Vejoris, Spain } \\
\text { Ankara, Turkey }\end{array}$ & $\begin{array}{l}{[37]} \\
{[30]} \\
{[43]} \\
{[39]}\end{array}$ \\
\hline Gnomoniopsis castaneae $^{*}$ & $\begin{array}{l}\text { several plant parts } \\
\text { bark, flower, leaf } \\
\text { several plant parts } \\
\text { flower, leaf, stem } \\
\text { shoot } \\
\text { fruit } \\
\text { buds } \\
\text { shoot } \\
\text { several plant parts } \\
\text { leaf or gall } \\
\text { leaf } \\
\text { leaf } \\
\text { branch }\end{array}$ & $\begin{array}{l}\text { Eurobin and Monbulk, Australia } \\
\text { several locations in New Zealand } \\
\text { Cuneo province, Italy } \\
\text { Southern Australia } \\
\text { several locations in Northern Italy } \\
\text { several locations in Switzerland } \\
\text { Aosta Valley and Piedmont, Italy } \\
\text { Geneve and Ticino, Switzerland } \\
\text { Monti Cimini, Italy } \\
\text { Southern Tuscany, Italy } \\
\text { Vejoris, Spain } \\
\text { Netherlands } \\
\text { Astroni Nature Reserve, Italy }\end{array}$ & $\begin{array}{c}{[37]} \\
{[29]} \\
{[49,50]} \\
{[51]} \\
{[52]} \\
{[53]} \\
{[54]} \\
{[38,41]} \\
{[44,55]} \\
{[30]} \\
{[43]} \\
{[56]} \\
{[47]}\end{array}$ \\
\hline Hyphodermella rosae & bark & Ismailly and Shaki, Azerbaijan & [33] \\
\hline Hypoxylon fragiforme & shoot (phellem) & Bellinzona and Murg, Switzerland & {$[28]$} \\
\hline Irpex lacteus & bark & Balakan, Azerbaijan & [33] \\
\hline Jattaea sp. & bark & Zagatala, Azerbaijan & {$[33]$} \\
\hline Massarina cf. quercina & shoot (phellem) & Murg, Switzerland & {$[28]$} \\
\hline $\begin{array}{c}\text { Mollisia sp. (= Cystodendron } \\
\text { sp.) }\end{array}$ & shoot (phellem) & Bellinzona, Switzerland & {$[28,57]$} \\
\hline Monodictys castaneae & shoot (phellem) & Bellinzona, Switzerland & {$[28]$} \\
\hline Mucor fragilis & bark & Vinhais, Portugal & [40] \\
\hline Neocucurbitaria cava* & leaf or gall & Southern Tuscany, Italy & [30] \\
\hline Neopestalotiopsis sp. & bark & Asturias, Spain & [35] \\
\hline
\end{tabular}


Table 1. Cont.

\begin{tabular}{|c|c|c|c|}
\hline Endophyte $^{1}$ & Plant Part & Country & Reference \\
\hline Neopestalotiopsis zimbabwana & bark & Asturias, Spain & [35] \\
\hline Nigrospora sp. & several plant parts & Eurobin and Monbulk, Australia & [37] \\
\hline Ophiovalsa cf. suffusa & shoot (lenticel) & Bellinzona, Switzerland & [28] \\
\hline Paraconiothyrium brasiliense & $\begin{array}{l}\text { bark } \\
\text { branch }\end{array}$ & $\begin{array}{c}\text { Vinhais, Portugal } \\
\text { Astroni Nature Reserve, Italy }\end{array}$ & $\begin{array}{l}{[40]} \\
{[47]}\end{array}$ \\
\hline Penicillium glabrum & bark & Vinhais, Portugal & {$[40]$} \\
\hline Penicillium sp. & $\begin{array}{l}\text { several plant parts } \\
\text { stem } \\
\text { bark } \\
\text { bud } \\
\text { bark } \\
\text { branch }\end{array}$ & $\begin{array}{c}\text { Eurobin and Monbulk, Australia } \\
\text { Black Sea region, Turkey } \\
\text { Marche, Italy } \\
\text { Ankara, Turkey } \\
\text { Valpaços, Portugal } \\
\text { Astroni Nature Reserve, Italy }\end{array}$ & $\begin{array}{l}{[37]} \\
{[31]} \\
{[34]} \\
{[39]} \\
{[40]} \\
{[47]}\end{array}$ \\
\hline Pestalotiopsis sp. & $\begin{array}{l}\text { leaf } \\
\text { bark } \\
\text { bud }\end{array}$ & $\begin{array}{l}\text { Vejoris, Spain } \\
\text { Asturias, Spain } \\
\text { Ankara, Turkey }\end{array}$ & $\begin{array}{l}{[43]} \\
{[35]} \\
{[39]}\end{array}$ \\
\hline Pezicula cinnamomea & shoot (phellem) & Bellinzona and Murg, Switzerland & [28] \\
\hline Phaeococcus sp. & shoot (phellem) & Murg, Switzerland & [28] \\
\hline Phoma sp. & $\begin{array}{l}\text { shoot (phellem) } \\
\text { several plant parts } \\
\text { bud }\end{array}$ & $\begin{array}{c}\text { Bellinzona, Switzerland } \\
\text { Eurobin and Monbulk, Australia } \\
\text { Ankara, Turkey }\end{array}$ & $\begin{array}{l}{[28]} \\
{[37]} \\
{[39]}\end{array}$ \\
\hline Pilidiella castaneicola * & shoot & Bellinzona and Murg, Switzerland & [28] \\
\hline Ramichloridium sp. & shoot (phellem) & Murg, Switzerland & [28] \\
\hline Rhizoctonia sp. & shoot (phellem) & Bellinzona, Switzerland & [28] \\
\hline Rhizopus sp. & bark & Vinhais, Portugal & {$[40]$} \\
\hline Sclerotinia pseudotuberosa & bark, bud, fruit & Viterbo province, Italy & [58] \\
\hline Sordaria rabenhorstii ${ }^{*}$ & bark & Valpaços, Portugal & {$[40]$} \\
\hline Sordaria sp. & $\begin{array}{l}\text { several plant parts } \\
\text { leaf or gall }\end{array}$ & $\begin{array}{l}\text { Eurobin and Monbulk, Australia } \\
\text { Southern Tuscany, Italy }\end{array}$ & $\begin{array}{l}{[37]} \\
{[30]}\end{array}$ \\
\hline Stemphylium vesicarium & leaf or gall & Southern Tuscany, Italy & [30] \\
\hline Trichoderma atroviride & $\begin{array}{l}\text { scion } \\
\text { leaf }\end{array}$ & $\begin{array}{l}\text { Ticino, Switzerland } \\
\text { Vejoris, Spain }\end{array}$ & $\begin{array}{l}{[38]} \\
{[43]}\end{array}$ \\
\hline Trichoderma hamatum & shoot & Ticino, Switzerland & [38] \\
\hline Trichoderma koningiopsis & bark & Shaki, Azerbaijan & [33] \\
\hline Trichoderma sp. & $\begin{array}{l}\text { stem } \\
\text { bark } \\
\text { bark }\end{array}$ & $\begin{array}{c}\text { Black Sea region, Turkey } \\
\text { Qakh, Azerbaijan } \\
\text { Marche, Italy }\end{array}$ & $\begin{array}{l}{[31]} \\
{[33]} \\
{[34]}\end{array}$ \\
\hline Trichothecium roseum & leaf or gall & Southern Tuscany, Italy & {$[30]$} \\
\hline Umbelopsis isabellina & $\begin{array}{l}\text { bark } \\
\text { bark }\end{array}$ & $\begin{array}{c}\text { Balakan and Qabala, Azerbaijan } \\
\text { Vinhais, Portugal }\end{array}$ & $\begin{array}{l}{[33]} \\
{[40]}\end{array}$ \\
\hline Xenoacremonium falcatum & bark & Balakan and Qabala, Azerbaijan & {$[33]$} \\
\hline Xylaria sp. & branch & Astroni Nature Reserve, Italy & [47] \\
\hline \multicolumn{4}{|c|}{ Castanea crenata } \\
\hline Alternaria sp. & leaf & Kashiwa, Japan & [59] \\
\hline Astrocystis sp. & leaf & Kashiwa, Japan & [59] \\
\hline Aureobasidium sp. & leaf & Kashiwa, Japan & [59] \\
\hline
\end{tabular}


Table 1. Cont.

\begin{tabular}{|c|c|c|c|}
\hline Endophyte $^{1}$ & Plant Part & Country & Reference \\
\hline Botryosphaeria dothidea & leaf & Kashiwa, Japan & [59] \\
\hline Colletotrichum acutatum & leaf & Kashiwa, Japan & [59] \\
\hline Colletotrichum gloeosporioides & leaf & Kashiwa, Japan & [59] \\
\hline Diaporthe sp. & leaf & Kashiwa, Japan & [59] \\
\hline Discula sp. & leaf & Kashiwa, Japan & [59] \\
\hline Glomerella sp. & leaf & Kashiwa, Japan & [59] \\
\hline Gnomoniopsis castaneae * & bark, flower, leaf & several locations in New Zealand & [29] \\
\hline Induratia fengyangensis * & leaf & Kashiwa, Japan & [59] \\
\hline Nigrospora sp. & leaf & Kashiwa, Japan & [59] \\
\hline Pestalotiopsis sp. & leaf & Kashiwa, Japan & {$[59]$} \\
\hline Phyllosticta capitalensis & leaf & Kashiwa, Japan & [59] \\
\hline Xylaria sp. & leaf & Kashiwa, Japan & [59] \\
\hline \multicolumn{4}{|c|}{ Castanea dentata } \\
\hline Acremonium implicatum * & stem & Michigan and Wisconsin, USA & [36] \\
\hline Alternaria alternata & stem & Michigan and Wisconsin, USA & [36] \\
\hline Alternaria brassicae & stem & Michigan and Wisconsin, USA & [36] \\
\hline Aspergillus tubingensis & stem & Michigan, USA & [60] \\
\hline Biscogniauxia aff. mediterranea & stem & $\begin{array}{c}\text { Michigan and North Carolina, } \\
\text { USA }\end{array}$ & {$[60]$} \\
\hline Botryosphaeria sp. & stem & Michigan and Wisconsin, USA & {$[32,36]$} \\
\hline Daldinia aff. childiae & stem & Michigan, USA & {$[60]$} \\
\hline Didimostylbe sp. & stem & Wisconsin, USA & {$[32]$} \\
\hline Diplodia corticola & stem & Michigan and Wisconsin, USA & {$[36]$} \\
\hline Diplodia seriata & stem & Michigan and Wisconsin, USA & [36] \\
\hline Dothiorella sp. & stem & Wisconsin, USA & {$[32]$} \\
\hline \multirow{3}{*}{ Epicoccum nigrum } & stem & Wisconsin, USA & {$[32]$} \\
\hline & stem & Michigan, USA & {$[60]$} \\
\hline & stem & Michigan and Wisconsin, USA & {$[61]$} \\
\hline Fusarium sp. & stem & Massachusetts, USA & {$[62]$} \\
\hline \multirow{2}{*}{ Gnomoniopsis castaneae $^{*}$} & flower, leaf & Ohaupo, New Zealand & [29] \\
\hline & stem & Michigan and Wisconsin, USA & {$[36]$} \\
\hline Mucor circinelloides & stem & Michigan and Wisconsin, USA & {$[36]$} \\
\hline Mucor fragilis & stem & Michigan and Wisconsin, USA & {$[36]$} \\
\hline Nectria cinnabarina & stem & Michigan and Wisconsin, USA & {$[36]$} \\
\hline Nigrospora aff. oryzae & stem & Michigan, USA & {$[60]$} \\
\hline Paraconiothyrium sp. & stem & Wisconsin, USA & {$[32]$} \\
\hline Penicillium glabrum & stem & Michigan and Wisconsin, USA & {$[32,36]$} \\
\hline Penicillium spinulosum & stem & Michigan and Wisconsin, USA & {$[32,36]$} \\
\hline Pestalotia sp. & stem & Wisconsin, USA & {$[31]$} \\
\hline Pestalotiopsis sp. & stem & North Carolina, USA & {$[60]$} \\
\hline
\end{tabular}


Table 1. Cont.

\begin{tabular}{|c|c|c|c|}
\hline Endophyte $^{1}$ & Plant Part & Country & Reference \\
\hline Pezicula cinnamomea & stem & Michigan and Wisconsin, USA & [36] \\
\hline Pezicula ericae & stem & Michigan and Wisconsin, USA & [36] \\
\hline Pezicula sporulosa & stem & North Carolina, USA & [60] \\
\hline Strasseria sp. & stem & Michigan and Wisconsin, USA & [36] \\
\hline Trichoderma atroviride & stem & Michigan and Wisconsin, USA & {$[32,36]$} \\
\hline Trichoderma aureoviride & stem & Wisconsin, USA & [32] \\
\hline Trichoderma citrinoviride & stem & Michigan and Wisconsin, USA & [36] \\
\hline Trichoderma harzianum & stem & Michigan and Wisconsin, USA & {$[36]$} \\
\hline Trichoderma sp. & stem & Massachusetts, USA & {$[62]$} \\
\hline Tubakia suttoniana * & stem & North Carolina, USA & [59] \\
\hline Umbelopsis isabellina & stem & Michigan and Wisconsin, USA & {$[32,36,61]$} \\
\hline \multicolumn{4}{|c|}{ Castanea mollissima } \\
\hline Alternaria eichhorniae & leaf & Qing Long, China & {$[63]$} \\
\hline Alternaria sp. & leaf & Qing Long, China & [63] \\
\hline Auriculibuller fuscus * & leaf & Qing Long, China & [63] \\
\hline Cercospora canescens & twig & Qing Long, China & {$[63]$} \\
\hline Cercospora sp. & twig & Qing Long, China & [63] \\
\hline Colacogloea sp. & leaf & Qing Long, China & [63] \\
\hline Colacogloea terpenoidalis & leaf & Qing Long, China & {$[63]$} \\
\hline Gnomoniopsis castaneae * $^{*}$ & bark, leaf & Ohaupo, New Zealand & [29] \\
\hline Kondoa sorbi & leaf & Qing Long, China & [63] \\
\hline Kondoa sp. & twig & Qing Long, China & {$[63]$} \\
\hline Papiliotrema sp. & leaf & Qing Long, China & [63] \\
\hline Phlebia acerina & - & China & [64] \\
\hline Sporobolomyces roseus & twig & Qing Long, China & [63] \\
\hline
\end{tabular}

${ }^{1}$ Underlined species are also known as disease agents of chestnuts. ${ }^{*}$ Names with an asterisk are the latest accepted for these species, which are different from the ones used in the corresponding references.

\subsection{Endophytic Fungi as Plant Disease Agents}

Chestnut is affected by many fungal pathogens, which sometimes have caused epidemics endangering this plant in more or less extended geographic contexts. Particularly, the blight and the ink diseases, respectively, caused by Cryphonectria parasitica and Phytophthora cinnamomi or P. cambivora, have required large-scale management with the concomitant employment of breeding, agronomic and biological control measures [65-69]. As known for many other plant pathogens, these fungi may present a latent stage during the disease cycle which can imply their possible recovery from asymptomatic tissues. In fact, recent investigations on microbial symbionts of several plant species are disclosing cases where fungal pathogens may persist in the endophytic condition for prolonged periods [70-72], supporting the hypothesis that at least some strains could behave as true endophytes in the absence of factors stimulating their pathogenicity. In the case of $C$. sativa, this aspect has been proposed as a possible explanation for the recovery of C. parasitica from asymptomatic coppice shoots of C. sativa in Switzerland [28]. This finding was followed by another report from France where the fungus could be re-isolated from asymptomatic tissues for up to seven months after artificial inoculation [45]. Although molecular methods have been developed for the detection of $C$. parasitica in plant tissues by real-time 
PCR $[73,74]$, to the best of our knowledge no systematic investigations have been dedicated to the eventual endophytic spread of this fungus.

The occasional endophytic occurrence of the chestnut blight agent must not be confused with the well documented case of hypovirulent strains. In fact, although they do not cause major damage to the plants, the concept of hypovirulence basically reflects the establishment of a pathogenic interaction [69]. On the other hand, it is also to be considered that hypovirulent strains can be recovered from healed cankers in plants where they had been experimentally inoculated, even at a certain distance from the previously symptomatic tissues [40]. Considering that the family Cryphonectriaceae mainly includes species with an endophytic lifestyle [75,76], the spread of hypovirulent strains within chestnuts, either after inoculation or in consequence of natural dispersal represents a case study deserving to be thoroughly analysed in view of a more formal assessment of how considering these border line ecological interactions. Indeed, recent observations seem to support the conjecture that mycoviruses can directly interact with the genome of fungi and convert necrotrophic pathogens into unharmful or even beneficial endophytes [77,78]. Moreover, additional data have pointed out that the pathogenic transition in C. parasitica was probably driven by the loss of genes involved in carbohydrate metabolism [79].

The additional fungal species known as pathogens of chestnuts that have been reported to occur as endophytes are underlined in Table 1. Some species are occasionally reported to cause dieback and cankers, such as Coryneum modonium (= Melanconia modonia) [80], Diplodina castaneae (= Sirococcus castaneae), better known as the agent of the Javart disease of chestnut [48], and Dendrostoma castaneum [28,46]. The latter represents a new name for Amphiporthe castanea (Diaporthales, Erythogloeaceae) [81], known to be widespread in Europe on C. sativa; this species is probably vertically transmitted as it has been found to infect seeds before harvest [46]. The recent description of several additional Dendrostoma species associated with Castanea spp. in Europe and China [81,82] calls for further assessments of the real nature of these symbiotic relationships. Botryosphaeria dothidea has been found to cause stem cankers and black rot of nuts on C. crenata, respectively, in Korea [83] and Japan [84,85]; moreover, on C. sativa it has been reported from cankers in the Black Sea region of Turkey [31] and from rotted nuts in Croatia, along with Diaporthe eres [86]. In China, the latter is also known to cause a brown margin leaf blight of C. mollissima [87]. Other fungi which damage fruits are Sclerotinia pseudotuberosa (= Ciboria batschiana), causing brown rot of nuts [52], Colletotrichum acutatum reported as the agent of a pink rot [88], Trichothecium roseum, Fusarium oxysporum, Botrytis cinerea, species of Penicillium, Aspergillus and Mucor and the above cited D. castaneum, whose incidence in determining moldy nuts is basically a secondary effect to erosions caused by moth and weevil larvae $[46,89]$. Conversely, so far there are no reports concerning the endophytic occurrence of the leaf spot agent Mycosphaerella maculiformis [44] or Fistulina hepatica which causes discoloration and red stain of chestnut wood [90].

Last but not the least, Gnomoniopsis castaneae has recently become the hottest fungal associate of chestnuts, being able to establish various types of interaction. This species was typified by Visentin et al. [50] quite recently, after having been described as Gnomonia pascoe (= Discula pascoe) from chestnut standings near Cuneo (Italy), displaying an endophytic behavior in several plant parts but causing disease symptoms on ripened fruits [49]. It now seems quite clear that this species was already known in taxonomy since 1879 with the name Phoma endogena [91]; later on, several independent reports used the names Phomopsis endogena, Phomopsis castanea and Phomopsis viterbensis, which are now considered as synonyms based on pictures and descriptions, even if a direct comparison of cultures is no more possible [52]. As previously mentioned for D. castaneum, the species is reported to be vertically transmitted [37]. Although sometimes the fungus was also associated to cankers, most of the old reports concern infection of nuts. Moreover, it has been described as agent of leaf and shoot blight and more recently reported to cause twig canker in Europe and India $[41,92]$. 


\subsection{Endophytic Fungi as Mutualists}

Besides having a direct effect on the pathogen, hypovirulent strains of C. parasitica can influence the course of chestnut blight by interfering with the development of endophytes. In this respect, an investigation carried out in Portugal showed that, with the single exception of Penicillium glabrum, endophytic fungi were less abundant in and around healed cankers after the treatment with hypovirulent strains [40]. Interactions between C. parasitica and endophytic fungi are important and deserve to be investigated more in depth. In fact, it has been inferred that, as a general feature, the impact of their antagonistic properties is higher against the hypovirulent than the virulent strains, which implies that they could impair the effectiveness of biological control of chestnut blights by negatively interfering with the natural spread of the former $[36,40]$.

In addition to some species also known as pathogens, such as G. castaneae and C. acutatum, with a potential antagonistic role against the blight agent [93], several species in Table 1 are reported to be involved at some extent in plant defense against pest and pathogens, thus representing examples of the ecological symbiotic relationship described as defensive mutualism [94]. This list includes Epicoccum nigrum, Fusarium lateritium and Paraconiothyrium brasiliense, but also species of Aspergillus, Chaetomium, Penicillium and Pestalotiopsis are frequently mentioned in investigations concerning this issue, particularly with reference to the production of bioactive compounds [95-98]. However, in this respect the most effective role is probably played by species of Trichoderma, representing the most considered antagonists of plant pathogenic fungi even in terms of practical employment in biological control $[99,100]$. Several species of this genus have been isolated from both C. sativa and $C$. dentata, with some indications of a direct involvement in protection against C. parasitica. In particular, T. atroviride was reported for endophytic occurrence in healthy leaves of C. sativa [43] and found to be abundant in healing cankers on C. dentata along with T. aureoviride [32]. Antagonistic effects by this species have been documented against G. castaneae on grafting scions [38]. Moreover, some trials on chestnut plants demonstrated effectiveness by Trichoderma strains in reducing blight symptoms [34,62,101]. Finally, in other studies the evaluation of antagonistic properties against $C$. parasitica has been limited to in vitro assays; effectiveness at some extent was observed for strains of Pezicula cinnamomea [28], Trichoderma spp., Penicillium sp., Gnomoniopsis sp., E. nigrum and Umbelopsis isabellina [61], Penicillium spp. [34] and Neopestalotiopsis zimbabwana [35].

\subsection{Endophytic Fungi and the Cynipid Gall Wasps}

As introduced above, fungi reported to occur in galls caused by the wasp Dryocosmus kuriphilus (Hymenoptera: Cynipidae) [102] are not to be considered as endophytes in the strict sense, since they develop in altered plant tissues. Nevertheless, many investigations carried out on this biological adversity have considered the possible role of fungi associated with these insect structures and examined their occurrence as endophytes.

The real nature of interactions between cynipid wasps and fungi is still debated. Systematic associations have been ascertained for other insects forming galls, such as midges belonging to the Asphondyliinae which are constantly associated with the cosmopolitan B. dothidea on whatever host plants based on a trophic relationship [103]. In the case of D. kuriphilus, indications are not univocal. An investigation carried out in Switzerland showed the existence of a possible link between galls and increased spread of chestnut blight. In fact, abandoned galls were found to be frequently colonized by virulent $C$. parasitica and considered to possibly represent the starting points of new infections; at the same time, the association appeared to be more relevant in stands where the insect was resident since longer time [93]. However, in a dedicated study the blight agent was not found on the body of wasps emerging from galls, which seems to rule out their possible role as vectors. Nevertheless, the finding of some endophytes, such as G. castaneae, C. acutatum and E. nigrum, represents an indication of the ability of wasps to act as their vectors, with possible effects on chestnut fitness [104]. Interestingly, the same species were isolated from galls in a Spanish study [43] and again in the course of the previously mentioned 
Swiss investigation [93], calling for their role in the tripartite relationships with plant and insects to be investigated more in depth. Besides the three above species, the latter study reports a long series of gall-associated fungi, many of which also known as endophytes of chestnuts (Table 1), including A. alternata, A. pullulans, B. mediterranea, B. dothidea, B. cinerea, F. oxysporum, H. rosae, N. cava, N. oryzae, P. brasiliense, P. glabrum, T. citrinoviride and T. harzianum.

Another mentioned pathogen/endophyte of chestnut, D. castaneae, was diffusely isolated from necrotic cynipid galls from several locations in Azerbaijan and Switzerland [48]. Indeed, gall necrosis is thought to severely affect vitality of $D$. kuriphilus, basically impacting the adults inside the galls. Investigations specifically concerning $G$. castaneae showed an exponential increase in gall necrosis during the season, reaching $75.4 \%$ in the mid of July. This process, which is likely to be triggered by resident endophytic inoculum, may result in an efficient control of the cynipid in chestnut stands; nevertheless, the high virulence to fruits is assumed to preclude use of this fungus in biocontrol strategies [55].

However, the issue of the relationships between cynipid wasps and G. castaneae remains quite controversial [56]. In fact, in another study the fungus could never be isolated from the insects, suggesting unlikeliness that D. kuriphilus may act as a vector of viable inoculum. The fungus was present in $33.8 \%$ of the buds before oviposition, while no association was detected between fungal colonization and oviposition. Moreover, the number of emerging adults was significantly higher from galls colonized by G. castaneae than from non-colonized ones, indicating a possible fungus/pest synergy. These findings suggest that this symbiotic association is asymmetrically favorable to the pest, and that it is eventually established after oviposition [54].

\section{Conclusions}

As for other crop species, our knowledge of the actual impact of endophytic fungi on chestnut fitness and economic performances is still at a preliminary stage. The extent at which research in the field may result in clearer evidence, and practical applications basically rely on the capacity to attain to more accurate assessments of the species assemblages in the different climatic and phytosanitary conditions. As an example, a recent study demonstrated that the yellow crinkle disease pathogen (Candidatus-phytoplasma castaneae) significantly changed the structure of fungal communities in chestnut leaves and twigs [63]. However, this analysis based on taxonomic aggregates higher than the species level has quite a limited significance, considering that higher taxa are inclusive of entities with very different ecological roles and impact. Moreover, another recent investigation on fungal rhizobiomes of chestnuts [105] tried to distinguish the species assortment according to the alleged guilds as inferable from categorization proposed in [106]. Based on our previous considerations, particularly on the possible shift endophyte/pathogen which is documented for many fungal species, such attempts appear to be not so useful for investigations aiming at establishing the ecological role played by a certain species and their eventual exploitation in crop management.

More in depth investigations are also required to better understand the nature of processes leading to the endophyte/pathogen conversion, also with reference to genetic diversity in Castanea [107]. Besides the above-mentioned role by mycoviruses, this biological phenomenon may also derive by the ability to produce phytotoxins or other compounds involved in disease induction. No investigations have been carried out so far on the production of secondary metabolites by endophytic fungi of chestnuts, with a single exception concerning a strain of $G$. castanea reported to produce abscisic acid and its diol derivative, which are possibly involved in pathogenicity [108].

Author Contributions: Conceptualization, R.N. and C.D.V.; resources, R.N. and G.L.B.; writingoriginal draft preparation, R.N., G.L.B., A.S. and C.D.V.; writing-review and editing, R.N., and C.C. All authors have read and agreed to the published version of the manuscript.

Funding: This research received no external funding. 
Institutional Review Board Statement: Not applicable.

Informed Consent Statement: Not applicable.

Data Availability Statement: Not applicable.

Conflicts of Interest: The authors declare no conflict of interest.

\section{References}

1. Manos, P.S.; Zhou, Z.K.; Cannon, C.H. Systematics of Fagaceae: Phylogenetic tests of reproductive trait evolution. Int. J. Plant Sci. 2001, 162, 1361-1379. [CrossRef]

2. Bounous, G. Il Castagno: Risorsa Multifunzionale in Italia e nel Mondo; Edagricole: Milano, Italy, 2014; p. 420. ISBN 9788850654151.

3. Beccaro, G.; Alma, A.; Bounous, G.; Gomes-Laranjo, J. The chestnut handbook. In Crop E Forest Management; Taylor \& Francis, CRC Press: Boca Raton, FL, USA, 2019; p. 378. ISBN 9780429445606.

4. Beccaro, G.L.; Donno, D.; Lione, G.G.; De Biaggi, M.; Gamba, G.; Rapalino, S.; Riondato, I.; Gonthier, P.; Mellano, M.G. Castanea spp. Agrobiodiversity conservation: Genotype influence on chemical and sensorial traits of cultivars grown on the same clonal rootstock. Foods 2020, 9, 1062. [CrossRef] [PubMed]

5. Pereira-Lorenzo, S.; Ballester, A.; Corredoira, E.; Vieitez, A.M.; Agnanostakis, S.; Costa, R.; Bounous, G.; Botta, R.; Beccaro, G.L.; Kubisiak, T.L. Chestnut. In Fruit Breeding; Springer: New York, NY, USA, 2012; pp. 729-769.

6. Bounous, G.; Marinoni, D.T. Chestnut: Botany, horticulture, and utilization. Hort. Rev. 2005, 31, $291-347$.

7. Mota, M.; Marques, T.; Pinto, T.; Raimundo, F.; Borges, A.; Caço, J.; Gomes-Laranjo, J. Relating plant and soil water content to encourage smart watering in chestnut trees. Agric. Water Manag. 2018, 203, 30-36. [CrossRef]

8. Warmund, M.R. Nutrient status and fruiting response of young Chinese chestnut trees following application of nitrogen. J. Am. Pomol. Soc. 2018, 72, 12-20.

9. Mellano, M.G.; Beccaro, G.L.; Donno, D.; Torello Marinoni, D.; Boccacci, P.; Canterino, S.; Cerutti, A.K.; Bounous, G. Castanea spp. Biodiversity conservation: Collection and characterization of the genetic diversity of an endangered species. Gen. Res. Crop Evol. 2012, 59, 1727-1741. [CrossRef]

10. Aryal, P.; Meiners, S.J.; Carlsward, B.S. Ectomycorrhizae determine chestnut seedling growth and drought response. Agrofor. Syst. 2020. [CrossRef]

11. Baptista, P.; Martins, A.; Pais, M.S.; Tavares, R.M.; Lino-Neto, T. Involvement of reactive oxygen species during early stages of ectomycorrhiza establishment between Castanea sativa and Pisolithus tinctorius. Mycorrhiza 2007, 17, 185-193. [CrossRef]

12. Baptista, P.; Martins, A.; Tavares, R.M.; Lino-Neto, T. Diversity and fruiting pattern of macrofungi associated with chestnut (Castanea sativa) in the Trás-os-Montes region (Northeast Portugal). Fungal Ecol. 2010, 3, 9-19. [CrossRef]

13. Reis, F.; Pereira, E.; Tavares, M.; Baptista, P.; Lino-Neto, T. Fungal community in chestnut orchards with different Hypholoma fasciculare above-ground abundance: Potential implications for sustainable production. Rev. Ciências Agr. 2016, 40, 124-132. [CrossRef]

14. Twieg, B.D.; Durall, D.M.; Simard, S.W. Ectomycorrhizal fungal succession in mixed temperate forests. New Phytol. 2007, 176, 437-447. [CrossRef]

15. Itoo, Z.; Reshi, Z. Influence of ectomycorrhizal inoculation on Pinus wallichiana and Cedrus deodara seedlings under nursery conditions. Front. Biol. 2014, 9, 82-88. [CrossRef]

16. Martins, A.; Marques, G.; Borges, O.; Portela, E.; Lousada, J.; Raimundo, F.; Madeira, M. Management of chestnut plantations for a multifunctional land use under Mediterranean conditions: Effects on productivity and sustainability. Agrofor. Syst. 2011, 81, 175-189. [CrossRef]

17. Stephenson, S.L.; Ali, M.B.H.B.; Rollins, A.W.; Furches, M.S.; Atherton, K.R. Ectomycorrhizal fungi associated with American chestnut at a site in Tennessee, USA. Castanea 2017, 82, 2-7. [CrossRef]

18. Álvarez-Lafuente, A.; Benito-Matías, L.F.; Peñuelas-Rubira, J.L.; Suz, L.M. Multi-cropping edible truffles and sweet chestnuts: Production of high-quality Castanea sativa seedlings inoculated with Tuber aestivum, its ecotype T. uncinatum, T. brumale, and T. macrosporum. Mycorrhiza 2018, 28, 29-38. [CrossRef] [PubMed]

19. Palmer, J.M.; Lindner, D.L.; Volk, T.J. Ectomycorrhizal characterization of an American chestnut (Castanea dentata) dominated community in Western Wisconsin. Mycorrhiza 2008, 19, 27-36. [CrossRef] [PubMed]

20. Santolamazza-Carbone, S.; Iglesias-Bernabé, L.; Sinde-Stompel, E.; Gallego, P.P. Ectomycorrhizal fungal community structure in a young orchard of grafted and ungrafted hybrid chestnut saplings. Mycorrhiza 2021. [CrossRef] [PubMed]

21. Gauthier, M.-M.; Zellers, K.E.; Löf, M.; Jacobs, D.F. Inter- and intra-specific competitiveness of plantation-grown American chestnut (Castanea dentata). For. Ecol. Manag. 2013, 291, 289-299. [CrossRef]

22. Fernández-Lorenzo, J.L.; Crecente, S. In vivo serial micrografting of Castanea sativa in short cycles. Acta Hortic. 2010, 866, 291-296. [CrossRef]

23. Coughlin, E.M.; Shefferson, R.P.; Clark, S.L.; Wurzburger, N. Plant-soil feedbacks and the introduction of Castanea (chestnut) hybrids to eastern North American forests. Restor. Ecol. 2020. [CrossRef]

24. Bauman, J.M.; Francino, S.; Santas, A. Interactions between ectomycorrhizal fungi and chestnut blight (Cryphonectria parasitica) on American chestnut (Castanea dentata) used in coal mine restoration. AIMS Microbiol. 2018, 4, 104-122. [CrossRef] 
25. Blom, J.M.; Vannini, A.; Vettraino, A.M.; Hale, M.D.; Godbold, D.L. Ectomycorrhizal community structure in a healthy and a Phytophthora-infected chestnut (Castanea sativa Mill.) stand in central Italy. Mycorrhiza 2009, 20, 25-38. [CrossRef] [PubMed]

26. Brundrett, M. Diversity and classification of mycorrhizal associations. Biol. Rev. 2004, 79, 473-495. [CrossRef]

27. Hyde, K.D.; Soytong, K. The fungal endophyte dilemma. Fungal Div. 2008, 33, 163-173.

28. Bissegger, M.; Sieber, T.N. Assemblages of endophytic fungi in coppice shoots of Castanea sativa. Mycologia 1994, 86, 648-655. [CrossRef]

29. Wadia, K.D.R.; Klinac, D.; McNeil, D.L.; Osmonalieva, A.; Stewart, A.; Knowles, R.D. Occurrence of Phomopsis castanea as an endophyte in chestnut trees. N. Z. Plant Prot. 2000, 53, 133-137. [CrossRef]

30. Fernandez-Conradi, P.; Fort, T.; Castagneyrol, B.; Jactel, H.; Robin, C. Fungal endophyte communities differ between chestnut galls and surrounding foliar tissues. Fungal Ecol. 2019, 42, 100876. [CrossRef]

31. Akilli, S.; Katircioðlu, Y.Z.; Maden, S. Chestnut blight cankers in black sea region of Turkey. Acta Hortic. 2009, 815, $247-252$. [CrossRef]

32. Double, M.L.; Kolp, M.R.; Jarosz, A.M.; Davelos Baines, A.; Fulbright, D.W.; MacDonald, W.L. Fungi associated with hypovirulent cankers of differing ages on American chestnut. Acta Hortic. 2013, 1043, 57-65. [CrossRef]

33. Aghayeva, D.N.; Rigling, D.; Meyer, J.B.; Mustafabeyli, E. Diversity of fungi occurring in the bark of Castanea sativa in Azerbaijan. Acta Hortic. 2017, 1220, 79-86. [CrossRef]

34. Murolo, S.; Concas, J.; Romanazzi, G. Use of biocontrol agents as potential tools in the management of chestnut blight. Biol. Contr. 2019, 132, 102-109. [CrossRef]

35. González, A.J.; Estefanía, T. Strains of Neopestalotiopsis sp. are in vitro antagonists of Cryphonectria parasitica. Biol. Contr. 2020, 143, 104187. [CrossRef]

36. Kolp, M.; Double, M.L.; Fulbright, D.W.; MacDonald, W.L.; Jarosz, A.M. Spatial and temporal dynamics of the fungal community of chestnut blight cankers on American chestnut (Castanea dentata) in Michigan and Wisconsin. Fungal Ecol. 2020, 45, 100925. [CrossRef]

37. Washington, W.S.; Hood, V.; Stewart-Wade, S. Phomopsis castanea, a seed-borne endophyte in chestnut trees. Austr. J. Bot. 1999, 47, 77-84. [CrossRef]

38. Pasche, S.; Crovadore, J.; Pelleteret, P.; Jermini, M.; Mauch-Mani, B.; Oszako, T.; Lefort, F. Biological control of the latent pathogen Gnomoniopsis smithogylvyi in European chestnut grafting scions using Bacillus amyloliquefaciens and Trichoderma atroviride. Dendrobiology 2016, 75, 113-122. [CrossRef]

39. Akilli Şimşek, S.; Akyüz, B.; Katircioğlu, Y.Z.; Serdar, Ü.; Maden, S. Effectiveness and efficacy of superficial disinfectants to prevent mechanical transmission of Cryphonectria parasitica from chestnut scion woods. Europ. J. Plant Pathol. 2020, 159, 131-138. [CrossRef]

40. Coelho, V.; Nunes, L.; Gouveia, E. Short and long term efficacy and prevalence of Cryphonectria parasitica hypovirulent strains released as biocontrol agents of chestnut blight. Eur. J. Plant Pathol. 2021. [CrossRef]

41. Pasche, S.; Calmin, G.; Auderset, G.; Crovadore, J.; Pelleteret, P.; Mauch-Mani, B.; Barja, F.; Paul, B.; Jermini, M.; Lefort, F. Gnomoniopsis smithogilvyi causes chestnut canker symptoms in Castanea sativa shoots in Switzerland. Fungal Genet. Biol. 2016, 87, 9-21. [CrossRef]

42. Haemmerli, U.A.; Brändle, U.E.; Petrini, O.; McDermott, J.M. Differentiation of isolates of Discula umbrinella (teleomorph: Apiognomonia errabunda) from beech, chestnut, and oak using randomly amplified polymorphic DNA markers. Mol. Plant-Microbe Interact. 1992, 5, 479-483. [CrossRef]

43. Muñoz-Adalia, E.J.; Rodríguez, D.; Casado, M.; Diez, J.; Fernández, M. Fungal community of necrotic and healthy galls in chestnut trees colonized by Dryocosmus kuriphilus (Hymenoptera, cynipidae). I-Forest 2019, 12, 411-417.

44. Vannini, A.; Morales-Rodriguez, C.; Aleandri, M.; Bruni, N.; Dalla Valle, M.; Mazzetto, T.; Martignoni, D.; Vettraino, A. Emerging new crown symptoms on Castanea sativa (Mill.): Attempting to model interactions among pests and fungal pathogens. Fungal Biol. 2018, 122, 911-917. [CrossRef] [PubMed]

45. Guérin, L.; Robin, C. Seasonal effect on infection and development of lesions caused by Cryphonectria parasitica in Castanea sativa. Forest Pathol. 2003, 33, 223-235. [CrossRef]

46. Sieber, T.N.; Jermini, M.; Conedera, M. Effects of the harvest method on the infestation of chestnuts (Castanea sativa) by insects and moulds. J. Phytopathol. 2007, 155, 497-504. [CrossRef]

47. Becchimanzi, A.; Nicoletti, R. Antagonistic relationships between Gnomoniopsis castaneae and endophytic fungi of chestnut (Castanea sativa). Antagonistic. Manuscript in preparation.

48. Meyer, J.B.; Trapiello, E.; Senn-Irlet, B.; Sieber, T.N.; Cornejo, C.; Aghayeva, D.; González, A.J.; Prospero, S. Phylogenetic and phenotypic characterisation of Sirococcus castaneae comb. nov. (synonym Diplodina castaneae), a fungal endophyte of European chestnut. Fungal Biol. 2017, 121, 625-637. [CrossRef] [PubMed]

49. Gentile, S.; Valentino, D.; Visentin, I.; Tamietti, G. An epidemic of Gnomonia pascoe on nuts of Castanea sativa in the Cuneo area. Acta Hortic. 2009, 866, 363-368. [CrossRef]

50. Visentin, I.; Gentile, S.; Valentino, D.; Gonthier, P.; Cardinale, F. Gnomoniopsis castanea sp. nov. (Gnomoniaceae, Diaporthales) as the causal agent of nut rot in sweet chestnut. J. Plant Pathol. 2012, 94, 411-419.

51. Shuttleworth, L.A.; Guest, D.I.; Liew, E.C.Y. Fungal planet description sheet 108-Gnomoniopsis smithogilvyi L.A. Shuttleworth, E.C.Y. Liew \& D.I. Guest, sp. nov. Persoonia 2012, 28, 142-143. 
52. Maresi, G.; Oliveira Longa, C.M.; Turchetti, T. Brown rot on nuts of Castanea sativa Mill: An emerging disease and its causal agent. iForest Biogeosci. For. 2013, 6, 294-301. [CrossRef]

53. Dennert, F.G.; Broggini, G.A.; Gessler, C.; Storari, M. Gnomoniopsis castanea is the main agent of chestnut nut rot in Switzerland. Phytopathol. Mediter. 2015, 54, 199-211.

54. Lione, G.; Giordano, L.; Ferracini, C.; Alma, A.; Gonthier, P. Testing ecological interactions between Gnomoniopsis castaneae and Dryocosmus kuriphilus. Acta Oecol. 2016, 77, 10-17. [CrossRef]

55. Vannini, A.; Vettraino, A.; Martignoni, D.; Morales-Rodriguez, C.; Contarini, M.; Caccia, R.; Paparatti, B.; Speranza, S. Does Gnomoniopsis castanea contribute to the natural biological control of chestnut gall wasp? Fungal Biol. 2017, 121, 44-52. [CrossRef] [PubMed]

56. Lione, G.; Danti, R.; Fernandez-Conradi, P.; Ferreira-Cardoso, J.V.; Lefort, F.; Marques, G.; Meyer, J.B.; Prospero, S.; Radocz, L.; Robin, C.; et al. The emerging pathogen of chestnut Gnomoniopsis castaneae: The challenge posed by a versatile fungus. Eur. J. Plant Pathol. 2019, 153, 671-685. [CrossRef]

57. Grünig, C.R.; Queloz, V.; Duò, A.; Sieber, T.N. Phylogeny of Phaeomollisia piceae gen. sp. nov.: A dark, septate, conifer-needle endophyte and its relationships to Phialocephala and Acephala. Mycol. Res. 2009, 113, 207-221. [CrossRef] [PubMed]

58. Vettraino, A.M.; Paolacci, A.; Vannini, A. Endophytism of Sclerotinia pseudotuberosa: PCR assay for specific detection in chestnut tissues. Mycol. Res. 2005, 109, 96-102. [CrossRef]

59. Matsumura, E.; Fukuda, K. A comparison of fungal endophytic community diversity in tree leaves of rural and urban temperate forests of Kanto district, eastern Japan. Fungal Biol. 2013, 117, 191-201. [CrossRef] [PubMed]

60. Potter, B. Biocontrol Potential of Endophytes of Healthy Castanea dentata Tissue for Application Against Cryphonectria parasitica. Master's Thesis, Univ. Wisconsin, La Crosse, WI, USA, 2017.

61. Kolp, M.; Fulbright, D.W.; Jarosz, A.M. Inhibition of virulent and hypovirulent Cryphonectria parasitica growth in dual culture by fungi commonly isolated from chestnut blight cankers. Fungal Biol. 2018, 122, 935-942. [CrossRef]

62. Tattar, T.A.; Berman, P.M.; González, E.Y.; Mount, M.S.; Dolloff, A.L. Biocontrol of the chestnut blight fungus Cryphonectria parasitica. Arboricult. J. 1996, 20, 449-469. [CrossRef]

63. Ren, F.; Dong, W.; Shi, S.; Dou, G.; Yan, D.H. Chinese chestnut yellow crinkle disease influence microbiota composition of chestnut trees. Microbial Pathogen. 2020, 152, 104606. [CrossRef]

64. Jia, X.; Zhu, X.; Luo, C.; Ye, Y.; Lu, D. Research of the B2 of endophytic fungi from Castanea millissima and its inhibition of fungi characteristics. J. Xinyang Normal Univ. Nat. Sci. 2011, 24, 209-214.

65. Milgroom, M.G.; Cortesi, P. Biological control of chestnut blight with hypovirulence: A critical analysis. Ann. Rev. Phytopathol. 2004, 42, 311-338. [CrossRef]

66. Turchetti, T.; Maresi, G. Biological control and management of chestnut diseases. In Integrated Management of Diseases Caused by Fungi, Phytoplasma and Bacteria; Springer: Dordrecht, The Netherlands, 2008; pp. 85-118.

67. Anagnostakis, S.L. Chestnut breeding in the United States for disease and insect resistance. Plant Dis. 2012, 96, 1392-1403. [CrossRef] [PubMed]

68. EFSA Panel on Plant Health (PLH). Scientific opinion on the pest categorisation of Cryphonectria parasitica (Murrill) Barr. EFSA J. 2014, 12, 3859. [CrossRef]

69. Rigling, D.; Prospero, S. Cryphonectria parasitica, the causal agent of chestnut blight: Invasion history, population biology and disease control. Mol. Plant Pathol. 2018, 19, 7-20. [CrossRef]

70. Nicoletti, R. Endophytic fungi of citrus plants. Agriculture 2019, 9, 247. [CrossRef]

71. Nicoletti, R.; Di Vaio, C.; Cirillo, C. Endophytic fungi of olive tree. Microorganisms 2020, 8, 1321. [CrossRef]

72. Salvatore, M.M.; Andolfi, A.; Nicoletti, R. The thin line between pathogenicity and endophytism: The case of Lasiodiplodia theobromae. Agriculture 2020, 10, 488. [CrossRef]

73. Rubio, S.; Barnes, A.; Webb, K.; Hodgetts, J. A real-time PCR assay for improved rapid, specific detection of Cryphonectria parasitica. Ann. Appl. Biol. 2017, 171, 52-61. [CrossRef]

74. Chandelier, A.; Massot, M.; Fabreguettes, O.; Gischer, F.; Teng, F.; Robin, C. Early detection of Cryphonectria parasitica by real-time PCR. Eur. J. Plant Pathol. 2019, 153, 29-46. [CrossRef]

75. Mausse-Sitoe, S.N.; Rodas, C.A.; Wingfield, M.J.; Chen, S.; Roux, J. Endophytic Cryphonectriaceae on native Myrtales: Possible origin of Chrysoporthe canker on plantation-grown Eucalyptus. Fungal Biol. 2016, 120, 827-835. [CrossRef] [PubMed]

76. Granados, G.M.; McTaggart, A.R.; Rodas, C.A.; Roux, J.; Wingfield, M.J. Species of Cryphonectriaceae occupy an endophytic niche in the Melastomataceae and are putative latent pathogens of Eucalyptus. Eur. J. Plant Pathol. 2020, 156, 273-283. [CrossRef]

77. Zhang, H.; Xie, J.; Fu, Y.; Cheng, J.; Qu, Z.; Zhao, Z.; Cheng, S.; Chen, T.; Li, B.; Wang, Q.; et al. A 2-kb mycovirus converts a pathogenic fungus into a beneficial endophyte for Brassica protection and yield enhancement. Mol. Plant 2020, 13, 1420-1433. [CrossRef]

78. Zhou, L.; Li, X.; Kotta-Loizou, I.; Dong, K.; Li, S.; Ni, D.; Wang, G.; Xu, W. A mycovirus modulates the endophytic and pathogenic traits of a plant associated fungus. ISME J. 2021. [CrossRef]

79. Stauber, L.; Prospero, S.; Croll, D. Comparative genomics analyses of lifestyle transitions at the origin of an invasive fungal pathogen in the genus Cryphonectria. Msphere 2020, 5, e00737-20. [CrossRef] [PubMed]

80. Adamčíková, K.; Juhásová, G.; Kobza, M.; Ondrušková, E. Diversity of microfungi on branches of Castanea sativa in Slovakia. Pol. Bot. J. 2013, 58, 741-746. [CrossRef] 
81. Jaklitsch, W.M.; Voglmayr, H. European species of Dendrostoma (Diaporthales). MycoKeys 2019, 59, 1. [CrossRef]

82. Jiang, N.; Fan, X.-L.; Crous, P.W.; Tian, C.-M. Species of Dendrostoma (Erythrogloeaceae, Diaporthales) associated with chestnut and oak canker diseases in China. MycoKeys 2019, 48, 67-96. [CrossRef] [PubMed]

83. Sung, J.M.; Han, S.S. Identification of canker-causing fungi associated with stems and twigs of chestnut tree. Korean J. Plant Pathol. 1986, 2, 174-184.

84. Hamasaki, K.; Kawaradani, M.; Shibao, M. Control of the chestnut weevil, Curculio sikkimensis (Heller), and the black rot fungus, Botryosphaeria dothidea (Mougeot) Cesati \& De Notaris, using hot water treatment. Ann. Rep. Kansai Plant Prot. Soc. 2016, $58,51-55$.

85. Nishimura, T. Study on fruit rot of Japanese chestnut. Bull. Hosei Univ. Graduate School. Graduate School Sci. Engin. 2015, 60, 13. [CrossRef]

86. Ivić, D.; Novak, A. Gljive povezane s truleži plodova pitomog kestena, s prvim nalazom Gnomoniopsis smithogilvyi u Hrvatskoj. Pomologia Croatica: Glasilo Hrvatskog Agronomskog Društva 2018, 22, 13-22. [CrossRef]

87. Gong, S.; Zhang, X.; Jiang, S.; Chen, C.; Ma, H.; Nie, Y. A new species of Ophiognomonia from Northern China inhabiting the lesions of chestnut leaves infected with Diaporthe eres. Mycol. Prog. 2017, 16, 83-91. [CrossRef]

88. Gaffuri, F.; Longa, C.M.O.; Turchetti, T.; Danti, R.; Maresi, G. ‘Pink rot': Infection of Castanea sativa fruits by Colletotrichum acutatum. Forest Pathol. 2017, 47, e12307. [CrossRef]

89. Donis González, I.R.; Fulbright, D.W.; Ryser, E.T.; Guyer, D. Shell mold and kernel decay of fresh chestnuts in Michigan. Acta Hortic. 2009, 866, 353-362. [CrossRef]

90. Yurkewich, J.I.; Castaño, C.; Colinas, C. Chestnut red stain: Identification of the fungi associated with the costly discolouration of Castanea sativa. Forest Pathol. 2017, 47, e12335. [CrossRef]

91. Spegazzini, C. Nova addenda ad Mycologiam Venetam. Michelia 1879, 1, 453-487.

92. Dar, M.A.; Rai, M.K. Gnomoniopsis smithogilvyi a canker causing pathogen on Castanea sativa: First report. Mycosphere 2015, 6, 327-336. [CrossRef]

93. Meyer, J.B.; Gallien, L.; Prospero, S. Interaction between two invasive organisms on the European chestnut: Does the chestnut blight fungus benefit from the presence of the gall wasp? FEMS Microbiol. Ecol. 2015, 91, fiv122. [CrossRef] [PubMed]

94. Saikkonen, K.; Saari, S.; Helander, M. Defensive mutualism between plants and endophytic fungi? Fungal Divers. 2010, 41, 101-113. [CrossRef]

95. Nicoletti, R.; Fiorentino, A.; Scognamiglio, M. Endophytism of Penicillium species in woody plants. Open Mycol. J. 2014, 8, 1-26. [CrossRef]

96. Fatima, N.; Muhammad, S.A.; Khan, I.; Qazi, M.A.; Shahzadi, I.; Mumtaz, A.; Hashmi, M.A.; Khan, A.K.; Ismail, T. Chaetomium endophytes: A repository of pharmacologically active metabolites. Acta Physiol. Plant. 2016, 38, 136. [CrossRef]

97. Deshmukh, S.K.; Prakash, V.; Ranjan, N. Recent advances in the discovery of bioactive metabolites from Pestalotiopsis. Phytochem. Rev. 2017, 16, 883-920. [CrossRef]

98. El-hawary, S.S.; Moawad, A.S.; Bahr, H.S.; Abdelmohsen, U.R.; Mohammed, R. Natural product diversity from the endophytic fungi of the genus Aspergillus. RSC Advan. 2020, 10, 22058-22079. [CrossRef]

99. Bailey, B.A.; Melnick, R.L. The endophytic Trichoderma. In Trichoderma: Biology and Applications; Mukherjee, P.K., Horwitz, B.A., Singh, U.S., Mukherjee, M., Schmoll, M., Eds.; CABI Publishing: Boston, MA, USA, 2013; pp. 152-172.

100. Rajani, P.; Rajasekaran, C.; Vasanthakumari, M.M.; Olsson, S.B.; Ravikanth, G.; Shaanker, R.U. Inhibition of plant pathogenic fungi by endophytic Trichoderma spp. through mycoparasitism and volatile organic compounds. Microbiol. Res. 2021, $242,126595$. [CrossRef]

101. Akilli, S.; Katircioğlu, Y.Z.; Maden, S. Biological control of chestnut canker, caused by Cryphonectria parasitica, by antagonistic organisms and hypovirulent isolates. Turkish J. Agric. For. 2011, 35, 515-523.

102. Cooper, W.R.; Rieske, L.K. Gall structure affects ecological associations of Dryocosmus kuriphilus (Hymenoptera: Cynipidae). Environ. Entomol. 2010, 39, 787-797. [CrossRef] [PubMed]

103. Zimowska, B.; Okoń, S.; Becchimanzi, A.; Krol, E.D.; Nicoletti, R. Phylogenetic characterization of Botryosphaeria strains associated with Asphondylia galls on species of Lamiaceae. Diversity 2020, 12, 41. [CrossRef]

104. Morales-Rodriguez, C.; Sferrazza, I.; Aleandri, M.; Dalla Valle, M.; Mazzetto, T.; Speranza, S.; Contarini, M.; Vannini, A. Fungal community associated with adults of the chestnut gall wasp Dryocosmus kuriphilus after emergence from galls: Taxonomy and functional ecology. Fungal Biol. 2019, 123, 905-912. [CrossRef]

105. Reazin, C.; Baird, R.; Clark, S.; Jumpponen, A. Chestnuts bred for blight resistance depart nursery with distinct fungal rhizobiomes. Mycorrhiza 2019, 29, 313-324. [CrossRef]

106. Nguyen, N.H.; Song, Z.; Bater, S.; Branco, S.; Tedersoo, L.; Menke, J.; Schilling, J.S.; Kennedy, P.G. FUNGuild: An open annotation tool for parsing fungal community datasets by ecological guild. Fungal Ecol. 2016, 20, 241-248. [CrossRef]

107. Nunziata, A.; Ruggieri, V.; Petriccione, M.; De Masi, L. Single nucleotide polymorphisms as practical molecular tools to support European chestnut agrobiodiversity management. Int. J. Mol. Sci. 2020, 21, 4805. [CrossRef] [PubMed]

108. Vinale, F.; Ruocco, M.; Manganiello, G.; Guerrieri, E.; Bernardo, U.; Mazzei, P.; Piccolo, A.; Sannino, F.; Caira, S.; Woo, S.L.; et al. Metabolites produced by Gnomoniopsis castanea [sic] associated with necrosis of chestnut galls. Chem. Biol. Technol. Agric. 2014, 6, 294-301. 